extrinsic mechanism. Overall, this study represents the first identification of Myd88 as a regulator of adult tracheal epithelial cell phenotype.

\section{Advances in screening and diagnosis of TB S38 COMMUNITY-BASED EVALUATION OF IMMIGRANT TB
SCREENING USING INTERFERON GAMMA RELEASE
ASSAYS AND TUBERCULIN SKIN TESTING: YIELDS AND
COST-EFFECTIVENESS}

doi:10.1136/thoraxjnl-2011-201054b.38

${ }^{1} \mathrm{M}$ Pareek, ${ }^{2} \mathrm{M}$ Bond, ${ }^{2} \mathrm{~J}$ Shorey, ${ }^{3} \mathrm{~S}$ Seneviratne, ${ }^{1} \mathrm{~A}$ Lalvani, ${ }^{2} \mathrm{O} \mathrm{M}$ Kon. ${ }^{1}$ Tuberculosis Research Unit, Imperial College London, London, UK; ${ }^{2}$ Tuberculosis Service, Chest and Allergy Clinic, St Mary's Hospital, Imperial College Healthcare NHS Trust, London, UK; ${ }^{3}$ Clinical Immunology, St Mary's Hospital, Imperial College Healthcare NHS Trust, London, UK

Background Tuberculosis (TB) notifications in the UK continue to rise due to disease in the foreign-born immigrant population. UK guidelines on immigrant screening have recently been revised but accurate calculation of cost-effectiveness is hampered by a lack of empiric data on the comparative performance of tuberculin skin test (TST) and interferon- $\gamma$ release-assays (IGRA) in immigrants arriving from countries with varying TB incidence.

Methods Prospective evaluation of TST and two commercially available IGRAs (QuantiFERON Gold in-tube (OFN-GIT) and TSPOT.TB) in recent immigrants aged $=16$ years to quantify test positivity, concordance and factors associated with a positive result for all three tests. We computed yields at different incidence thresholds and the relative cost-effectiveness, using a decisionanalysis-model stratified by HIV/drug-resistance, of screening using different latent TB infection (LTBI) screening modalities at varying incidence thresholds supplemented with/without port-of-arrival chest radiography.

Results 231 immigrants included; median age 29 (IOR 24-37). TST accepted by $80.9 \%$, read in $93.6 \% ; 30.3 \%$ positive. OFN-GIT and TSPOT.TB positive in $16.6 \%$ and $22.5 \%$ respectively. Positive TST, OFN-GIT and T-SPOT.TB independently associated with increasing TB incidence in immigrants' countries of origin ( $p=0.008,0.007$ and 0.01 respectively). Implementing current guidance (depending on test) would identify $98 \%-100 \%$ of LTBI but also require $97 \%-99 \%$ of the immigrant cohort to be tested; raising the threshold to 150/ 100000 (includes immigrants from Indian Subcontinent) would identify $49 \%-71 \%$ of LTBI but require half the cohort to be screened. The three most cost-effective screening strategies (which were more cost-effective than current guidance) were: no CXR at port-of-entry and screen with single-step OFN-GIT at 250/100 000 (Incremental cost-effective ratio (ICER) $£ 21565.3 /$ per case averted), no CXR at port-of-entry and screen with single-step OFN-GIT at 150/100000 (averted additional 7.8 cases of active TB, ICER of $£ 31867.1 /$ per case averted) and no CXR at port-of-entry and screen with single-step OFN-GIT at 40/100 000 which averted a further 9.4 cases (ICER £34 753.5/per case averted).

Conclusions Immigrant screening in the UK could cost-effectively and safely eliminate mandatory CXR on arrival by emphasising systematic screening for LTBI with single-step IGRA. An intermediate incidence threshold for screening balances the need to identify as much imported LTBI as possible against limited service capacity.

\section{S39 MIGRATION AND TUBERCULOSIS: THE START OF INTELLIGENT NEW ENTRANTS SCREENING}

doi:10.1136/thoraxjnl-2011-201054b.39

${ }^{1} \mathrm{M}$ E Kruijshaar, ${ }^{2} \mathrm{M}$ Lipman, ${ }^{3} \mathrm{~J}$ Moore, ${ }^{1} \mathrm{I}$ Abubakar. ${ }^{1}$ Health Protection Agency, London, UK; ${ }^{2}$ Royal Free Hospital, London, UK; ${ }^{3}$ London School of Hygiene and Tropical Medicine, London, UK

Tuberculosis (TB) remains a problem in the UK, and almost threequarters of active TB cases occur in the non-UK born. Most of these are likely infected abroad and strategies to detect latent TB in this population are being considered. We investigated how soon after arrival into the UK certain groups developed TB and the implications of this for numbers needed to screen and treat. Numbers of migrants arriving in 2005 from the top 6 countries of origin (of TB cases) were obtained from the Labour Force Survey (LFS). National TB surveillance (ETS) provided information on active cases from these countries. Estimates of interferon $\gamma$ release assay (IGRA) positive cases $(20 \%-28 \%)$, IGRA sensitivity $(84 \%)$, and efficacy $(65 \%)$ and completeness $(85 \%)$ of chemoprophylaxis were obtained from Pareek et al. (Lancet ID 2011). The Abstract S39 table 1 shows numbers needed to screen and treat to prevent one case of TB developing in the UK in the 5 years after arrival. Numbers were relatively low, especially for Bangladesh and Somalia. The Abstract S39 table 1 also shows time between diagnosis and entry into the UK, which varied between countries of the Indian Subcontinent and sub-Saharan Africa and changed over time. While $45 \%$ of cases born in India had been in the country more than 10 years prior to arrival in 2000-2004, this was $32 \%$ in $2005-2009$. Conversely, $57 \%$ of cases born in Zimbabwe were diagnosed within 2 years of arrival in 2000-2004, decreasing to $15 \%$ in $2005-2009$. The relatively low numbers needed to treat among migrants from these high burden countries provide support for new guidance to expand latent infection treatment. The observed trends and differences in time since entry reflect underlying migration patterns, with higher but decreasing levels of migration from sub-Saharan Africa and an established and ongoing

Abstract S39 Table 1 Estimated numbers needed to screen and treat to prevent a TB case in the 5 years after arrival, and time since entry into the UK of TB cases, England Wales and Northern Ireland

\begin{tabular}{|c|c|c|c|c|c|c|c|c|c|c|c|}
\hline \multirow{3}{*}{$\begin{array}{l}\text { Country of } \\
\text { birth-top } 6\end{array}$} & \multicolumn{5}{|c|}{ Estimated numbers needed to screen and treat, based on immigration in 2005} & \multicolumn{6}{|c|}{ Time between diagnosis and arrival into the UK of TB cases reported in: } \\
\hline & \multirow{2}{*}{$\begin{array}{l}\text { Immigrants } \\
\text { in } 2005^{*}\end{array}$} & \multicolumn{2}{|c|}{ TB cases $2005-2009$} & \multicolumn{2}{|c|}{ Number per case } & \multicolumn{3}{|c|}{$2000-2004$} & \multicolumn{3}{|c|}{$2005-2009$} \\
\hline & & All & Entry $2005 \dagger$ & Screen & Treat & $0-1$ year & $2-9$ years & $10+$ years & $0-1$ year & $2-9$ years & $10+$ years \\
\hline India & 41337 & 6403 & 546 & 163 & 33 & $25 \%$ & $30 \%$ & $45 \%$ & $26 \%$ & $42 \%$ & $32 \%$ \\
\hline Somalia & 10156 & 2883 & 293 & 75 & 21 & $34 \%$ & $53 \%$ & $13 \%$ & $24 \%$ & $57 \%$ & $19 \%$ \\
\hline Pakistan & 15533 & 4336 & 285 & 117 & 23 & $22 \%$ & $32 \%$ & $46 \%$ & $20 \%$ & $37 \%$ & $43 \%$ \\
\hline Bangladesh & 3058 & 1123 & 101 & 65 & 13 & $24 \%$ & $31 \%$ & $45 \%$ & $21 \%$ & $38 \%$ & $41 \%$ \\
\hline Zimbabwe & & 1097 & 67 & 0 & 0 & $57 \%$ & $40 \%$ & $3 \%$ & $15 \%$ & $78 \%$ & $7 \%$ \\
\hline Nigeria & 14578 & 810 & 72 & 436 & 122 & $38 \%$ & $37 \%$ & $25 \%$ & $28 \%$ & $52 \%$ & $20 \%$ \\
\hline All countries & 405943 & 16652 & 2130 & 411 & 82 & $29 \%$ & $36 \%$ & $35 \%$ & $23 \%$ & $46 \%$ & $31 \%$ \\
\hline
\end{tabular}

*Population which entered the UK in 2005 that remain in the country as at Jan-Mar 2010 (LFS).

†Number of TB cases reported in 2005-2009 that were known to have entered the UK in 2005 (ETS). 
migrant population from the Indian Subcontinent. A more detailed analysis of migration patterns and its impact on UK cases of TB is warranted.

\section{S40 A CROSS SECTIONAL INVESTIGATION TO DETERMINE THE BACKGROUND PREVALENCE OF LATENT TUBERCULOSIS INFECTION IN UNSELECTED MEDICAL INPATIENTS IN A LOW PREVALENCE REGION OF UK REVEALS HIGH RATES OF IGRA POSITIVITY}

doi:10.1136/thoraxjnl-2011-201054b.40

${ }^{1} \mathrm{~N}$ Varsani, ${ }^{2} \mathrm{~T}$ S C Hinks, ${ }^{2} \mathrm{D}$ T Godsiff, ${ }^{2} \mathrm{~T}$ C Bull, ${ }^{3} \mathrm{~K}$ L Nash, ${ }^{3} \mathrm{~L}$ McLuckie, ${ }^{3} \mathrm{~A}$ Warley. ${ }^{1}$ St George's University of London, London, UK; ${ }^{2}$ Department of Infection, Inflammation and Immunity, University of Southampton School of Medicine, Southampton, UK; ${ }^{3}$ Salisbury NHS Foundation Trust, Salisbury, UK

Introduction The background rate of latent tuberculosis infection (LTBI) in low prevalence regions of the UK is unknown. Interferon $\gamma$ release assays (IGRAs) are sensitive and specific methods for detecting LTBI, and have accurately characterised the epidemiology of LTBI among high risk populations such as recent TB contacts or immigrants. However there are no current data on the incidence of IGRA positivity among the general adult population in the UK. Such data would be valuable for interpreting the significance of a positive IGRA result, and guiding cost-benefit analyses of new diagnostics. Methods A TB outbreak occurred within a rural DGH. 481 individuals were identified as potential contacts and were tested by IGRA

Abstract S40 Table 1

\begin{tabular}{|c|c|c|c|c|}
\hline & \multicolumn{4}{|c|}{ Unexposed, age-matched control patients } \\
\hline & $\begin{array}{l}\text { TSpot positive } \\
\mathrm{n}=13 \\
\mathrm{n}(\%)\end{array}$ & $\begin{array}{l}\text { TSpot negative } \\
\mathrm{n}=135 \\
\mathrm{n}(\%)\end{array}$ & OR & Univariate $\mathbf{p}$ \\
\hline Age (years), median (range) & $64(45-87)$ & $70(25-93)$ & & 0.81 \\
\hline Male, n (\%) & $5(38)$ & $70(52)$ & & 0.36 \\
\hline \multicolumn{5}{|l|}{ Ethnicity, n (\%) } \\
\hline White Caucasian & $13(100)$ & $135(100)$ & & \\
\hline \multicolumn{5}{|l|}{ Country of birth } \\
\hline UK born & $9(69)$ & $125(93)$ & 0.18 & 0.006 \\
\hline Other & $4(31)^{*}$ & $9(6.7)$ & & \\
\hline $\begin{array}{l}\text { Years since immigration, } \\
\text { median (range) }\end{array}$ & $43(21-62)$ & $52(8-61)$ & & \\
\hline $\begin{array}{l}\text { Ever visited a high prevalence } \\
\text { country, n (\%) }\end{array}$ & $4(31)$ & $46(37)$ & & 0.84 \\
\hline $\begin{array}{l}\text { Years since last visit, } \\
\text { median (range) }\end{array}$ & $8.5(0-40)$ & $5.5(0-60)$ & & \\
\hline $\begin{array}{l}\text { Ever resident in a high prevalence } \\
\text { area }>6 / 52, n(\%)\end{array}$ & $2(15)$ & $27(20)$ & & 0.64 \\
\hline $\begin{array}{l}\text { Occupation: healthcare, prison, } \\
\text { lab n }(\%) \dagger\end{array}$ & $4(31)$ & $18(13)$ & & 0.09 \\
\hline \multicolumn{5}{|l|}{ BCG (history or scar), $\mathrm{n}(\%)$} \\
\hline Yes & $7(54)$ & $66(49)$ & & 0.73 \\
\hline No & $4(31)$ & $44(33)$ & & \\
\hline Unknown & $2(15)$ & $25(19)$ & & \\
\hline \multicolumn{5}{|l|}{ Medical history, $\mathrm{n}(\%)$} \\
\hline $\begin{array}{l}\text { Comorbidity known to be } \\
\text { associated with TB } \neq\end{array}$ & $0(0)$ & $21(16)$ & & 0.22 \\
\hline Immunosuppresive medications§ & $4(31)$ & $12(9.2)$ & 4.6 & 0.015 \\
\hline Ever known to be exposed to TB & $4(31)$ & $26(19)$ & & 0.32 \\
\hline Ever had treatment for tuberculosis & $2(15)$ & $2(1.5)$ & 12 & 0.003 \\
\hline
\end{tabular}

*Germany (2), South Africa (1), Hong Kong (1).

†Percentages are those of those with valid data (eg occupation).

$\ddagger$ Diabetes mellitus (17), chronic renal failure (3), haematological malignancy (2), gastric surgery (1).

§Systemic steroids (12), methotrexate (4), hydroxychloroquine (2), anti-TNF- $\alpha$ (2), sulfasalzine (1).
(TSpot.TB). Uniquely, for comparison, we recruited an additional large cohort of age matched controls from the same general wards but with no exposure to the outbreak.

Results 456 staff and patients were tested including 148 unexposed age-matched patient controls. Rates of positivity were $22 \%(95 \% \mathrm{CI}$, 14 to 29 ), $11 \%$ (6.1 to 16 ), $8.8 \%$ (4.2 to 13 ) and $9.5 \%$ (3.0 to 22 ) among exposed patients, exposed staff, unexposed patients and unexposed staff respectively. 8 cases of active TB (identical VNTR profile) and an estimated 35 cases of recently acquired LTBI can be attributed to exposure to the index case, out of 481 contacts. Characteristics of the unexposed controls are in Abstract S40 table 1. IGRA positivity was associated in multivariate analyses with history of previous TB treatment ( $O R$ 11, $p=0.04$ ) and use of corticosteroids (OR 5.9, $\mathrm{p}=0.02$ ), but not with age. The age specific prevalences of IGRA positivity were 0 (N/A) for ages $<40,15.3 \%$ (12.2 to 29.4 ) for ages $40-59,7.0 \%$ (0.92 to $13 \%$ ) for age $60-79$, and $10 \%(5.9$ to 19$)$ for ages $=80$.

Conclusions We observed a surprisingly high background rate of IGRA positivity among an unselected population typical of respiratory and general medical inpatients in a rural DGH. All controls were white-Caucasians, who comprise $92 \%$ of the UK population, and may represent a current minimum UK background rate. As rates were highest in the 5th and 6th decade, in the context of ageing populations and increasing iatrogenic immunosuppression, reactivation of LTBI may be a persistent hazard for several decades to come.

\section{S41 SCREENING FOR LATENT TB IN HIV: ARE NICE \& BHIVA GUIDANCE EFFECTIVE?}

doi:10.1136/thoraxjnl-2011-201054b.41

S Capocci, C Smith, I Cropley, S Bhagani, M A Johnson, M C I Lipman. Royal Free Hospital, London, UK

HIV infection is the strongest single risk factor for the development of active TB in latently infected individuals. NICE and BHIVA 2011 guidelines both recommend screening for latent TB in HIV positive subjects in the UK. NICE bases this on blood CD4 count; and recommends testing all patients with $\mathrm{CD} 4<500$ cells/ $\mu \mathrm{l}$ (if CD4 $<200$, this is with an Interferon Gamma Release Assay (IGRA) and tuberculin skin test (TST), and if CD4 200-500, then IGRA \pm TST is proposed). BHIVA test-stratify on CD4 count, country of origin and use of antiretroviral therapy (ART). They recommend IGRA in the following populations: all subjects from Sub Saharan Africa, irrespective of CD4 if on ART $<2$ years; medium TB incidence countries if on ART $<2$ years and CD4 $<500$; and low TB incidence countries if on ART $<6$ months and CD $4<350$. To our knowledge, neither strategy has been formally tested in a UK HIV population. Here, using data on all subjects over a 10-year period (2000-2010) within our large HIV service ( $n=3306)$, we determine the impact of applying both strategies to detect cases of latent TB infection who subsequently developed active TB during this time period $(n=72)$. Subjects who developed active $T B<3$ months from HIV diagnosis were excluded from analysis, as it was felt that the

\section{Abstract S41 Table 1}

\begin{tabular}{lll}
\hline & NICE & BHIVA \\
\hline Eligible for screening & 2778 & 1478 \\
Number eligible, who developed TB (PPV) & $66(2.4 \%)$ & $42(2.8 \%)$ \\
Not eligible for screening & 528 & 1828 \\
Number not eligible, who developed TB & $6(1.1 \%)$ & $30(1.6 \%)$ \\
NPV & $98.9 \%$ & $98.4 \%$ \\
Sensitivity & $92 \%$ & $58 \%$ \\
Specificity & $16 \%$ & $56 \%$ \\
\hline
\end{tabular}

Note: PPV $=$ positive predictive value, NPV = negative predictive value 